\title{
Recombinant Neisseria surface protein A is a potential vaccine candidate against Neisseria meningitides serogroup B
}

\author{
SHANGYUN YING ${ }^{1 *}$, JUN HE $^{1,2^{*}}$, MINJUN YU ${ }^{1}$, YUKUAI ZHANG ${ }^{1}$, SUHONG DENG $^{1}$, \\ LUSI ZHANG $^{1}$, MEIHUA XIE ${ }^{1}$ and SIHAI HU ${ }^{1}$ \\ ${ }^{1}$ Pathogenic Biology Institute, College of Basic Medicine; ${ }^{2}$ Department of Clinical Laboratory, Nanhua Affiliated Hospital, \\ University of South China, Hengyang, Hunan 421001, P.R. China
}

Received September 24, 2013; Accepted April 14, 2014

DOI: $10.3892 / \mathrm{mmr} .2014 .2325$

\begin{abstract}
Neisseria meningitidis is the pathogen of epidemic encephalomyelitis and is responsible for permanent damage to the brain and nervous system. In the present study, the prokaryotic expression vector $\mathrm{pGEX}-6 \mathrm{p}-1 /$ neisseria surface protein A (NspA) was constructed and the immune protective effect was investigated with the purified recombinant $\mathrm{rNspA}$. Female BALB/c mice were immunized by intraperitoneal inoculation of rNspA, glutathione S-transferase (GST) or phosphate-buffered saline (PBS). The protection experiment in mice demonstrated that the protection rate of the rNspA group was $85 \%$ against the $N$. meningitidis strain MC58, and a serum bactericidal assay in vitro revealed that the serum bactericidal titer of the rNspA group reached 1:64 following three immunizations. The levels of specific immunoglobulin (Ig) A (SIgA), IgG, IgG1, IgG2a, IgG2b and IgG3 of mice in the rNspA group peaked at week six and were higher than those in the mice in the GST and PBS groups. The levels of stimulation index, interleukin-4 and interferon- $\gamma$ in the culture supernatant of the spleen lymphocytes of the rNspA group increased in a time-dependent manner and were higher than those of the mice in the GST and PBS groups over the same period. The results suggested that rNspA may induce increased specific humoral and cellular immune responses, and that it is effectively protective against $N$. meningitidis serogroup B in mice. The present study offered novel evidence that may lead to the development of a novel effective $N$. meningitidis serogroup B vaccine.
\end{abstract}

Correspondence to: Dr Sihai Hu, Pathogenic Biology Institute, College of Basic Medicine, University of South China, No. 28 West Changsheng Road, Hengyang, Hunan 421001, P.R. China

E-mail: hhsshh_518@163.com

*Contributed equally

Key words: Neisseria surface protein A, recombinant protein, vaccine candidate, protection, Neisseria meningitides serogroup B

\section{Introduction}

Neisseria meningitidis, the pathogen of epidemic cerebrospinal meningitis, induces permanent damage to the brain and nervous system $(1,2)$. This pathogen is also responsible for the development of invasive diseases caused by $N$. meningitidis, including septicemia, pneumonia and arthritis (3-5). Epidemic cerebrospinal meningitis has become a major public health problem, and developing novel methods to effectively control and prevent it has attracted worldwide attention.

Vaccinations are one of the major, most effective strategies for the control and prevention of diseases caused by $N$. meningitidis (4). According to the molecular structure and antigenicity of capsular polysaccharide, $N$. meningitidis is divided into 13 serogroups (A, B, C, D, H, I, K, L, X, Y, Z, 29E and W135), among which $N$. meningitidis serogroup $\mathrm{A}, \mathrm{B}$ and C cause $\leq 90 \%$ of meningitis cases $(6,7)$. At present, vaccines in specific to N. meningitidis serogroup A, C, Y and W135 based on capsular polysaccharides have been successively developed, which have been proved to effectively control the prevalence of epidemic cerebrospinal meningitis caused by the corresponding serogroup (8-10). However, recent studies have revealed that the probability of a significant increase in the prevalence of epidemic cerebrospinal meningitis caused by $N$. meningitidis serogroup B is high $(11,12)$. Despite the fact that capsular polysaccharide occurs on the surface of N. meningitidis serogroup B, it is likely to trigger a cross reaction, as its structure is similar to mammalian gangliosides. Therefore, vaccines that mimic the capsular polysaccharide of $N$. meningitidis serogroup B are not suitable for development due to the risk of autoimmune disease $(13,14)$.

It has been identified in recent years that Neisseria surface protein A (NspA) is a low molecular membrane protein that exists in the surface of all $N$. meningitidis and consists of 525 nucleotides, whose antigenicity is evolutionarily conservative (15). Protein crystal structure analysis demonstrated that NspA is composed of eight peptides with an antiparallel $\beta$-tubular structure among which, the annular extracellular section forms a relatively prolonged adhesion area. The area is mainly composed of hydrophobic residues and an anchored molecule, and therefore, it is hypothesized that NspA may functionally interact with hydrophobic materials (16). Despite the precise functioning of NspA remaining to be elucidated, it 
is known that its gene sequence is similar to the opacity (Opa) protein in the outer membrane protein family, and accordingly, it has the function of indirect adhesion to the host cells (17). Studies utilizing isotope-labeled anti-NspA monoclonal antibodies have confirmed that NspA is located on the surface of complete cells and is one of the most typical membrane proteins of $N$. meningitidis (18). Therefore, NspA is considered to be a candidate antigen for the development of a vaccine for epidemic cerebrospinal meningitis caused by $N$. meningitidis serogroup B.

In the present study, the constructed prokaryotic expression vector pGEX-6p-1/NspA was transfected into Escherichia (E.) coli BL21 to express the rNspA. Humoral immunity and cellular immunity levels induced by purified rNspA inoculated in $\mathrm{BALB} / \mathrm{c}$ mice were detected and the immunoprotective effect was evaluated to provide a vaccine candidate aganist N. meningitidis serogroup B.

\section{Materials and methods}

Materials. The DNA Ladder (1 Kb), BamHI, NotI and T4 ligase were purchased from NEB (Hitchin, UK). Protein Marker was purchased from Fermentas (Burlington, ON, Canada). Plasmid Miniprep kit and Polymerase Chain Reaction (PCR) Product Purification kit were purchased from Omega (Norcross, GA, USA). Glutathione S-transferase (GST) Purification resin was purchased from Merck KGaA (Darmstadt, Germany). ELISA kit was purchased from eBioscience. N. meningitidis Serogroup B Diagnostic antisera were purchased from BD Biosciences (Franklin Lakes, NJ, USA).

Strains, cultivation and experimental animals. pGEX-6p-1 vector, E. coli strain JM109, Escherichia coli strain BL21 were used as conventional recombinant experiments. $N$. meningitidis serogroup B strain MC58 was purchased from American Type Culture Collection (ATCC; Manassas, VA, USA). Four- to five-week old, specific-pathogen free female BALB/c mice and newborn rabbits were purchased from the Faculty of Experimental Animals at the University of South China (Hengyang, China). The animals were raised on a normal diet at $25^{\circ} \mathrm{C}$ and $50 \%$ humidity on a 12 -h light/dark cycle. The study was approved by the Ethics Committee of the University of South China (Hengyang, China).

Construction and identification of $p G E X-6 p-1 / N s p A$. BamHI and NotI were selected as the restriction sites for the upstream and downstream primers, respectively. The NspA fragment was amplified by PCR using the $N$. meningitidis serogroup B genomic DNA as templates and the primers 5'-CGGGATCCATGAAAAAAGCACTTGCCGCACTG-3' and 5'-TTGCGGCCCGCTAACCGCCGACAGTCGCTAC-3' were used. Following enzyme digestion and ligation, pGEX-6P-1/NspA was constructed. The construct was confirmed by restriction digestion and sequencing.

Expression, purification and identification of rNspA. pGEX-6P-1/NspA was transformed into E. coli BL21 and induced by isopropyl $\beta$-D-1-thiogalactopyranoside (IPTG). Following ultrasonication, the supernatant was collected and GST affinity chromatography purification was conducted to obtain purified recombinant NspA protein (rNspA). The rNspA protein was stored at $-70^{\circ} \mathrm{C}$ after its concentration was detected with the Bradford method. Mouse serum following rNspA immunization was used as the primary antibody and horseradish peroxidase-labeled sheep anti-mouse IgG (Beyotime Institute of Biotechnology, Haimen, China) was used as the secondary antibody. Western blot analysis was used to indentify rNspA.

Model construction of MC58 strain infection. A total of 40 female 9-10-week old BALB/c mice were randomly divided into four groups, with ten mice in each group. A $0.9 \% \mathrm{NaCl}$ solution was used to dilute the bacterial suspension. N. meningitidis strain MC58 suspension of optical density (OD) $\mathrm{A} 600=0.005$ (a concentration equivalent to $4,000 \mathrm{CFU} / \mathrm{ml}$ ) was immediately injected into the abdominal cavity of the mice. The control group was injected with phosphate-buffered saline (PBS) of the same amount. Within $72 \mathrm{~h}$ following the attack, the disease and survival rate of the mice was monitored. Peritoneal fluid in the dead mice and blood in the surviving mice were obtained to inoculate in chocolate agar plates (BD Biosciences) and within 12-24 h, the growth was observed. Colonies were identified by gram staining, biochemical and specificity PCR amplification.

Animal immunization and specimen collection. A total of 60 female BALB/c mice (4-5 weeks old) were selected and randomly divided into three groups, with 20 mice in each group. Immunization with $100 \mu \mathrm{g}$ rNspA (rNspA group), $100 \mu \mathrm{g}$ GST (GST group), PBS (control group) were administered intraperitoneally. Prior to each immunization, $50 \mu \mathrm{l}$ of Freund's incomplete adjuvant was added to the mixture, and immunization was performed every two weeks, for a total of three times. The mouse reproductive tract fluid and mouse tail venous blood were collected in week 0,2, 4, 6 and 8. Following immunization, a suspension of $N$. meningitidis strain MC58 bacteria was utilized to bacterially challenge the mice. Two weeks following the final immunization, the mice were dissected and the spleens were obtained. A 200 nylon gauze mesh was used for filtering to produce a cell suspension.

Serum bactericidal assay (SBA). The N. meningitidis strain MC58 suspension was mixed with rabbit complement (Pel-Freez Biologicals, Rogers, AR, USA) at a 1:1 ratio, the mixture was combined with the immune serum in a two-fold diluted concentration and cultured for $1 \mathrm{~h}$ at $37^{\circ} \mathrm{C}$. The chocolate agar plates were then inoculated and incubated at $37^{\circ} \mathrm{C}$ overnight. At the same time, the diagnostic sera of $N$. meningitidis serogroup B were used as a positive control and PBS as a negative control. The negative control included bacterial suspension plus complement, inactivated bacteria plus complement, and serum plus bacteria plus heating deactivation complement. Deactivation complement was considered as the reference. For the serum with a bactericidal rate $>50 \%$, the reciprocal of the highest dilution multiple was as bactericidal antibody titers of serum antibody.

Immune activity assay. rNspA as the antigen was coated on 96-well plates and vaginal lavage fluid of the mice was obtained. The indirect ELISA method was used to detect 

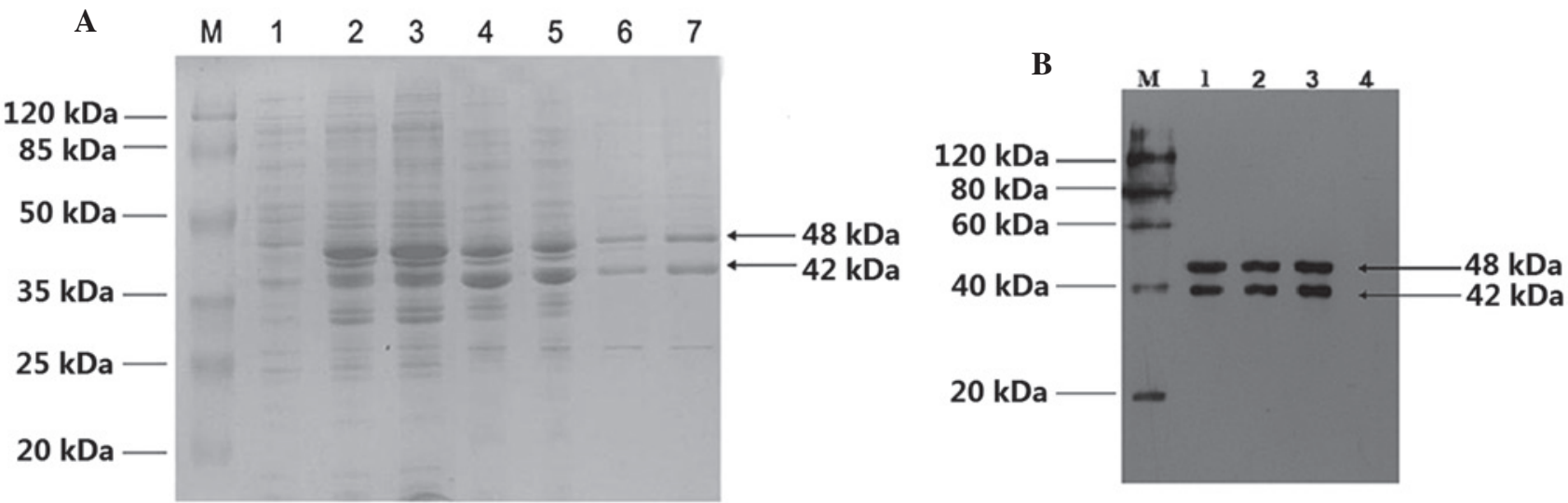

Figure 1. Expression, purification and identification of rNspA. (A) Expression and purification of rNspA from the cell supernatant. Band M, protein marker; band 1, supernatant of IPTG-induced BL21 cells; band 2-5, the supernatant of pGEX-6P-1/NspA-transfected BL21 cells induced by IPTG; band 6-7, purified rNspA by glutathione S-transferase resin. (B) Following western blot analysis of the purified rNspA, two strips with the molar weights of 42 and 48 kDa were observed. Band M, EasySee western protein marker; bands 1-3, purified rNspA; band 4, blank control. rNspA, recombinant neisseria surface protein A; GST, glutathione S-transferase; IPTG,. isopropyl $\beta$-D-1-thiogalactopyranoside.

specific immunoglobulin A (SIgA) levels induced by the rNspA. The serum of immune mice was collected and the indirect ELISA method was used to detect specific IgG, IgG1, IgG2a, IgG2b and IgG3 antibody levels. A total of $10 \mu \mathrm{g} \mathrm{rNspA}$ was used to stimulate spleen cells of the immune mice and an CCK- 8 colorimetric assay was used to detect the proliferation index of the spleen lymphocytes. Three days following cultivation, the cells were collected to perform lysis by ultrasonication. The indirect ELISA method was used to detect the levels of interferon (IFN) $-\gamma$ and interleukin (IL)-4.

Statistical analysis. All data are expressed as the mean \pm standard deviation. Independent sample t-tests were used to perform the comparison in pairs within the groups. Multiple groups were compared using repeated data variance t-test. Statistical analysis was performed using the statistical software program, SPSS, version 13.0 (SPSS, Inc., Chicago, IL, USA). P<0.05 was considered to indicate a statistically significant difference between values.

\section{Results}

Expression, purification and identification of $r N s p A$. The purity and native conformation of recombinant proteins is essential for the mice to be able to produce specific protective antibodies and to reduce non-specific interference. As demonstrated in Fig. 1A, the exploration consequences of induced expression demonstrated that the rNspA had the highest expression in the cell supernatant under the conditions of $30^{\circ} \mathrm{C}$ and $1 \mathrm{mmol} / \mathrm{l} \mathrm{IPTG}$. By purification of the GST resin, two proteins with molecular weights of 40 and $44 \mathrm{kDa}$, equal to the estimated values, were observed. As shown in Fig. 1B, western blot analysis with rNspA immune serum as the primary antibody identified two proteins close to $45 \mathrm{kDa}$. The results indicated that rNspA is effectively expressed in E. coli BL21.

Protective effects of rNSPA and serum bactericidal analysis. The BALB/c mouse model infected with the $N$. meningitidis

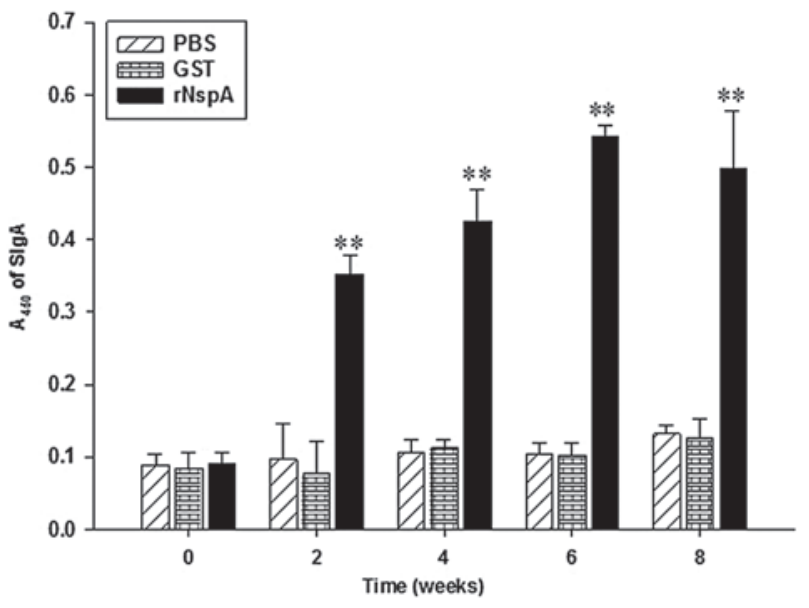

Figure 2. Detection of SIgA in mice immunized with rNspA. Vaginal secretions of the mice were obtained following $0,2,4,6$ and 8 weeks of immunization, then the indirect ELISA method was used for detection of SIgA levels. ${ }^{* *} \mathrm{P}<0.01$, comparison between the rNspA and PBS groups and between the NspA and GST groups demonstrated significant differences. Data are presented as the mean \pm standard deviation. The values were calculated from three independent experiments. rNspA, recombinant neisseria surface protein A; PBS, phosphate-buffered saline; GST, glutathione S-transferase; SIgA, specific immunoglobulin A.

strain MC58 was successfully constructed for the evaluation of specific protective effects induced by the candidate antigens. As demonstrated in Table I, at $72 \mathrm{~h}$, the protection rate of the rNspA group was $85 \%$, while that of the mice in the GST and PBS groups was $0 \%$. The results revealed that rNspA had significant protective effects on mice against the N. meningitidis strain MC58.

The SBA is internationally recommended as the gold standard for the evaluation of serological immune effects, which was used for the detection of functional antibodies with bactericidal activity in serum. As shown in Table I, following SBA method optimization, the serum bactericidal titer of rNspA group reached 1:64 following three immunizations; however, that of the GST group only reached 1:2 and that of the PBS group was 0 . The results indicated that $\mathrm{rNspA}$-induced immune 

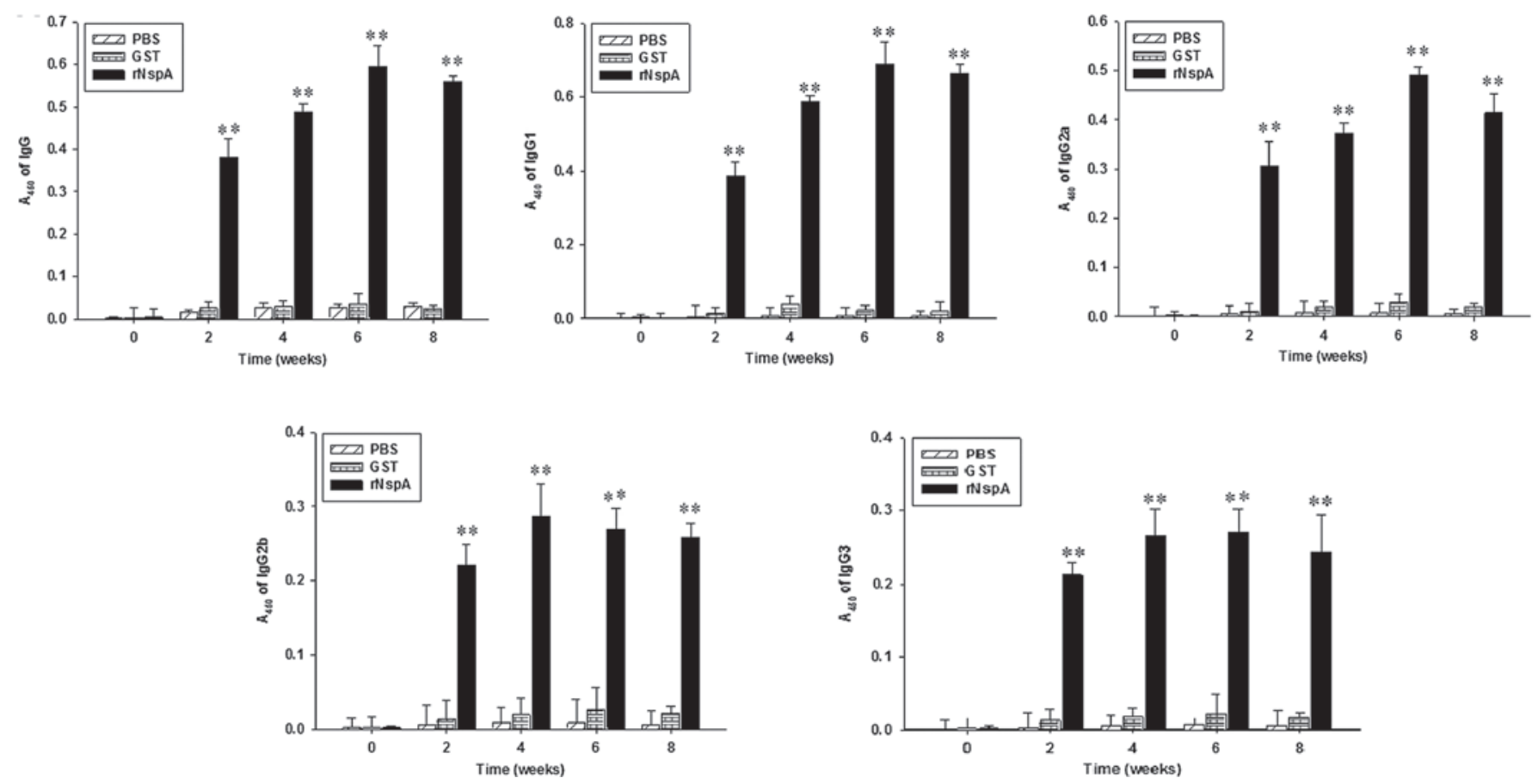

Figure 3. Levels of $\operatorname{IgG}$, IgG1, IgG2a, IgG2b and IgG3 in serum were determined after the mice were immunized with rNspA. The serum of mice was obtained $0,2,4,6$ and 8 weeks following immunization, then the indirect ELISA method was used for detection of IgG, IgG1, IgG2a, IgG2b and IgG3 levels. ${ }^{* *} \mathrm{P}<0.01$, for the comparisons between the rNspA and PBS groups and between the NspA and GST groups the results were significantly different. Data are presented as the mean \pm standard deviation. The values were calculated from three independent experiments. rNspA, recombinant neisseria surface protein A; PBS, phosphate-buffered saline; GST, glutathione S-transferase; Ig, immunoglobulin.

serum had a complement-dependent bactericidal effect in vitro and was highly protective in mice against the $N$. meningitidis strain MC58.

SIgA of mice immunized with $r N s p A$. SIgA levels were detected to determine whether the antigen may activate the mucosal immune system in mice. As revealed in Fig. 2, the levels of SIgA in the rNspA group demonstrated an upward trend with increasing time; however, the levels of SIgA decreased at week eight. At week six, following immunization, the levels of SIgA of mice in the rNspA group peaked and the antibody titer was $\leq 1: 2,800$. The levels of SIgA in the mice in the rNspA group were higher than those of the mice in the GST and PBS groups over the same time period, while no significant difference was identified between the mice in the GST and PBS groups. The results revealed that rNspA may induce high levels of mucosal immune responses in mice.

Humoral immunity in mice immunized with $r N s p A$. The total specific IgG reflected the response levels of mice on humoral immunity and that the types of cell-mediated immunity may be reflected by the IgG subclasses. As demonstrated in Fig. 3, the levels of IgG, IgG1 and IgG2a in the rNspA group exhibited an upward trend as time increased; however, the levels of $\mathrm{IgG}, \mathrm{IgG1}$ and $\mathrm{IgG} 2 \mathrm{a}$ decreased at week eight. At week six following immunization, the levels of $\mathrm{IgG}, \mathrm{IgG} 1$ and $\mathrm{IgG} 2 \mathrm{a}$ of the mice in the rNspA group peaked and the antibody titer was $1: 8,800,1: 6,400$ and 1:5,120, respectively. The levels of $\mathrm{IgG}, \mathrm{IgG1}, \mathrm{IgG} 2 \mathrm{a}$ in the mice in the rNspA group were higher than those in the mice in the GST and PBS groups over the same period, while no significant difference was identified

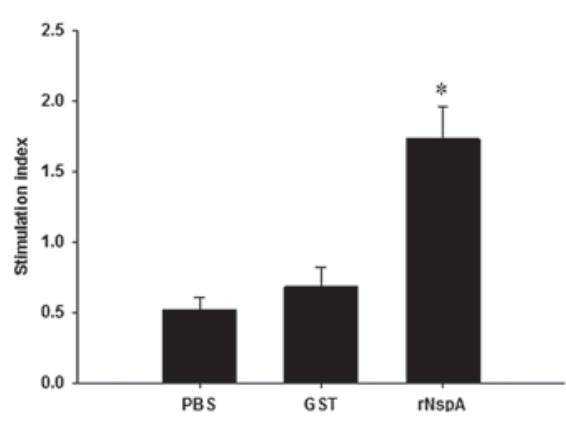

Figure 4. Detection of the spleen lymphocyte SI in mice. The spleen cells of mice were obtained two weeks following the final immunization and the CCK-8 colorimetric assay was used for determination of the proliferation index of simulated spleen lymphocytes of the mice in each group. ${ }^{* *} \mathrm{P}<0.05$, for the comparisons between the rNspA and PBS groups and between the NspA and GST groups the results were significantly different. Data are presented as the mean \pm standard deviation. The values were calculated from three independent experiments. SI, stimulation index; rNspA, recombinant neisseria surface protein A; PBS, phosphate-buffered saline; GST, glutathione S-transferase.

between the mice in the GST and PBS groups. The serum IgG2a/IgG1 ratios in the rNspA group at week 2, 4, 6 and 8 following immunization were $0.795(0.307 / 0.386), 0.637$ (0.373/0.586), $0.710(0.490 / 0.690)$ and $0.624(0.414 / 0.663)$, respectively. All ratios were $<1$. The results suggested that the rNspA-immunized mice produced a high level of humoral immune response, which was dominated by the Th2-type.

IgG2b and IgG3 have complement-mediated opsonization in the humoral immune response. As shown in Fig. 3D and E, the $\mathrm{IgG} 2 \mathrm{~b}$ antibody titers of the mice in the $\mathrm{rNspA}$ group reached a 
A

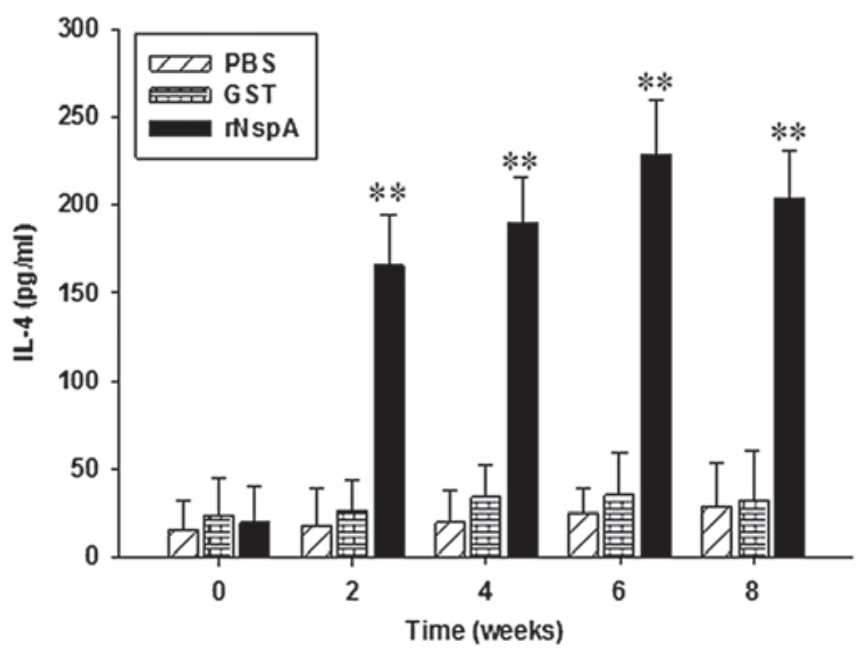

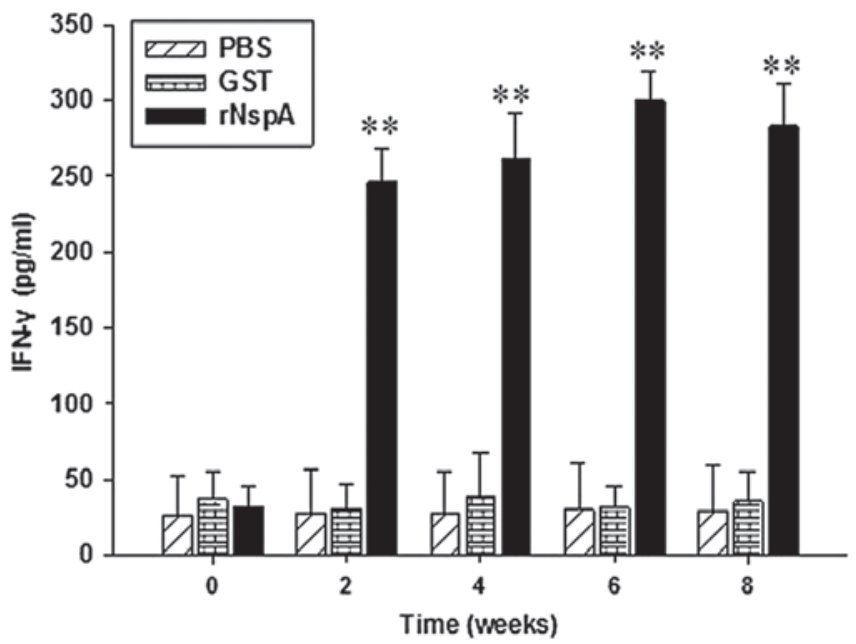

Figure 5. Detection of levels of IL-4 and IFN- $\gamma$ in spleen lymphocytes culture supernatant. The spleen cells of mice were obtained two weeks following the last immunization, then the indirect ELISA was used to detect the levels of IL-4 and IFN- $\gamma$ in the culture supernatant of spleen lymphocytes in each group. ${ }^{* *} \mathrm{P}<0.01$, for the comparisons between the rNspA and PBS groups and between the NspA and GST groups the results were significantly different. Data are presented as the mean \pm standard deviation. The values were calculated from three independent experiments. rNspA, recombinant neisseria surface protein A; PBS, phosphate-buffered saline; GST, glutathione S-transferase; IL, interleukin; IFN, interferon.

Table I. Immune protection evaluation of BALB/c mice after rNspA immunization.

\begin{tabular}{lcrrrr}
\hline & & \multicolumn{3}{c}{ Surviving mice $^{\mathrm{a}}(\mathrm{n})$} & \\
\cline { 3 - 4 } Groups & SBA & $24 \mathrm{~h}$ & $48 \mathrm{~h}$ & $\geq 72 \mathrm{~h}$ & Survival \% \\
\hline rNspA & $64^{* *}$ & $20 / 20$ & $20 / 20$ & $17 / 20$ & $85^{* *}$ \\
GST & 2 & $8 / 20$ & $0 / 20$ & $0 / 20$ & 0 \\
PBS & - & $6 / 20$ & $0 / 20$ & $0 / 20$ & 0
\end{tabular}

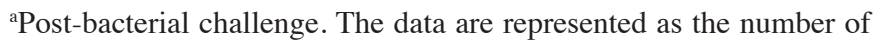
surviving mice in each group within $72 \mathrm{~h} .{ }^{* *} \mathrm{P}<0.01$, for the comparisons between the rNspA and PBS groups and between the NspA and GST groups the results were significantly different. Data are presented as the mean \pm standard deviation. The values were calculated from three independent experiments. rNspA, recombinant neisseria surface protein A; SBA, serum bactericidal assay; PBS, phosphate-buffered saline; GST, glutathione S-transferase.

peak $(1: 3,200)$ at week four. The IgG3 antibody titer of the mice in the rNspA group at week six reached a peak $(1: 2,800)$. The levels of $\operatorname{IgG} 2 \mathrm{~b}$ and $\operatorname{IgG} 3$ in the mice in the rNspA group were higher than those in the mice in the GST and PBS groups over the same period, while no significant difference was identified between the mice in the GST and PBS groups. These results suggested that the rNspA-immunized mice produced high levels of IgG2b and IgG3, which may mediate complementary activation and regulation so as to improve the immune defense against $N$. meningitidis.

Cellular immunity levels in mice immunized with $r$ NspA. The immune response state of the spleen lymphocytes in mice immunized with rNspA was determined through detection of the spleen lymphocyte stimulation index (SI). As illustrated in
Fig. 4, following stimulation of the spleen lymphocytes of the mice by rNspA, the SI value of the mice in the rNspA group $(1.61 \pm 0.04)$ was significantly higher than that of the mice in the GST $(0.63 \pm 0.01)$ and the PBS $(0.49 \pm 0.02)$ groups, while no significant difference was identified between the mice in the GST and PBS groups. The results suggested that the immune response of spleen lymphocytes may be activated in the mice immunized with rNspA.

Levels of IL-4 and IFN- $\gamma$ in the culture supernatant of spleen lymphocytes. IL-4 stimulates B-cell proliferation and is involved in the differentiation of Th2 cells in the body's immune response. IFN- $\gamma$ has immunomodulatory functions, which activate macrophages, thus producing cytotoxic effects and improving the activity and cytotoxicity of $\mathrm{T}$ lymphocytes. As demonstrated in Fig. 5, the levels of IL-4 and IFN- $\gamma$ in the culture supernatant of spleen lymphocytes in the rNspA group exhibited an upward trend as the time increased and at week six following immunization, the levels of IL-4 and IFN- $\gamma$ in the mice in the rNspA group reached a peak. The levels of IL-4 and IFN- $\gamma$ in the mice in the rNspA group were higher than those of the mice in the GST and PBS groups over the same period of time, while no significant difference was identified between the mice in the GST and PBS groups. The results revealed that the mice immunized with $\mathrm{rNspA}$ produced a certain level of cellular immune responses.

\section{Discussion}

$N$. meningitidis is the pathogen of epidemic encephalomyelitis and predominantly affects children between the ages of six months to two years, who are particularly susceptible due to their underdeveloped immune systems. The mortality rate is high following infection $(13,14)$. Currently, among all N.meningitidis groups, the capsular polysaccharide in $N$. meningitidis serogroup B has a similar structure to that of the human tissue, 
and therefore, a vaccine based on a capsular polysaccharide from $N$. meningitidis serogroup B may not be used clinically as it may trigger an autoimmune disease. Therefore, to date, an effective vaccine against $N$. meningitidis serogroup B remains to be developed $(3,4)$.

The establishment of an animal infection model with $N$. meningitidis serogroup B significantly facilitates the pathogenic study of $N$. meningitidis in a host and allows the evaluation of protective effects of specific candidate antigens (19). The pathogenic factors of $N$. meningitidis to the animal host include a capsule, fimbriae, immunoglobulin A1 protease and lipooligosaccharides (20). Mortality ensues following $N$. meningitidis infection in $\mathrm{BALB} / \mathrm{c}$ mice (21), and therefore, BALB/c mice were selected as an animal model for N. meningitidis MC58 infection in the present study. The mortality rates of the animals were observed four weeks following infection and these data were used for evaluation of the immunoprotective effects of rNspA in mice.

As a result of the rapid development of recombinant DNA technology and reverse volcanology, new candidate antigens for $N$. meningitidis serogroup B are being continuously discovered; in particular, studies have focused on outer membrane proteins $(22,23)$. NspA protein is a low molecular membrane protein that exists in the surface of all $N$. meningitidi. More importantly, NspA protein is highly conserved with stable antigenicity, which are optimal properties for a potential candidate vaccine antigen (16).

Previous studies have demonstrated that rNspA exists in different forms at different temperatures, e.g. $22 \mathrm{kDa}$ at $95^{\circ} \mathrm{C}$, 16 and $22 \mathrm{kDa}$ at $105^{\circ} \mathrm{C}$ and $16 \mathrm{kDa}$ at $125^{\circ} \mathrm{C}(23) \cdot \mathrm{rNspA}$ is a small protein and its fusion with the GST tag may improve its immunogenicity. In the present study, two distinct bands were identified when $\mathrm{rNspA}$ was either treated at $95^{\circ} \mathrm{C}$ or $105^{\circ} \mathrm{C}$, which may be explained by the temperature-dependent breakage of disulfide bonds. These results were consistent with the molecular size of rNspA reported by Martin et al (23).

Previous studies have revealed that rNspA may offer $80 \%$ protection against $N$. meningitidis strain $608 \mathrm{~B}$ and $100 \%$ protection against $N$. meningitidis strain 164B in mice (24). A study reported that rNspA may offer $80 \%$ protection against N. meningitidis strain H44/76 (25) and the present study revealed that it offered $85 \%$ protection against $N$. meningitidis serogroup B strain MC58. These findings suggested that rNspA induced protective immunity in mice and subsequently reduced mortality in mice. During an in vitro serum bactericidal test, it was identified that the serum immunized with rNspA exhibited bactericidal effects when compared with the GST and PBS immunized mice. The mortality rate of $N$. meningitidis strain MC58 was $>50 \%$ using the serum from primary immunized mice at a dilution ratio of 1:32 and following three immunizations. Serum at a dilution of 1:64 also eliminated 50\% of N. meningitidis MC58 under the mediation of a complement system.

Mucosa is the first line of defense against invading microbes and secretory IgA effectively neutralizes pathogens (26). The SIgA titer in the lavage fluid of the reproductive tract was as high as 1:2,800 at week six in the rNspA group, which indicated that rNspA induced efficient local mucosal immunity and provided mucosal protection. $N$. meningitidis colonizes in nasopharyngeal mucosa and effective mucosal immunity is important for the clearance of $N$. meningitidis $(27,28)$.
The serum IgG titer was as high as 1:8,800 following rNspA immunization in mice and a high level of specific IgG and its subclasses IgG1, IgG2a, IgG2b and IgG3 were detected, which indicated that rNspA may induce enhanced humoral immune responses. Several studies have demonstrated that $\mathrm{IgG} 2 \mathrm{a}$ in serum reflects the Th1 cellular immune response and IgG1 reflects the Th2 humoral immune response (29). Following three immunizations, the $\mathrm{IgG} 2 \mathrm{a} / \mathrm{IgG1}$ ratio in serum was always $<1$, which indicated the $\mathrm{rNspA}$-induced immune response was of the Th2 type. In the cell-mediated immune response, the rNspA group demonstrated an improved effect in activating spleen lymphocytes as compared with the GST and PBS groups. Th1 cells mainly secrete type I cytokines, including IFN $-\gamma$ and TNF- $\beta$, which facilitate Th0 differentiation towards Th1. Th2 cells mainly secrete type II cytokines, including IL-4 and IL-5, which facilitate Th0 differentiation towards Th2 (30). In the present study, high levels of IFN- $\gamma$ and IL-4 were detected in the supernatant of mice spleen lymphocytes. These results indicated that rNspA induced cellular immunity and humoral immunity (mainly Th2) that may have a useful protective effect in mice against the $N$. meningitidis strain MC58.

The complement binding site in Fc fragments of $\operatorname{IgG} 2 b$ and $\mathrm{IgG} 3$ is exposed once they are bound to rNspA, which binds Clq and strongly activates the activity of the complement $(31,32)$. As principal in vivo opsonin, the Fc fragment of IgG may bind to Fc receptors (FcRs) on the surface of phagocytes and FcR-bound IgG1 and IgG3 exhibit a strong opsonizing activity (33). High levels of specific IgG, IgG2a, IgG2b and IgG3 were detected in the rNspA group serum and high levels of IgG2b and IgG3 may activate the complement and enhance opsonization and a bactericidal effect (34). Furthermore, previously reported results demonstrated that rNspA, as an antigen, may induce complement-dependent bactericidal activity and enhance the body's resistance to microbe invasion $(24,26)$.

In conclusion, the present study demonstrated that rNspA induced higher and specific mucosal, humoral and cellular immune responses. Furthermore, rNspA-induced antibody regulated complement-dependent bactericidal activity and mediated resistance to the $N$. meningitidis strain MC58. The present study offers new evidence that may aid in the development of an effective $N$. meningitidis serogroup B vaccine and further study is required to investigate the potential clinical applications of these results.

\section{Acknowledgements}

This study was supported by the National Natural Science Foundation of China (no. 81172890), the Construct Program of the Key Discipline in Hunan Province and Hunan Provincial Key Laboratory for Special Pathogens Prevention and Control (no. 2014-5-2012-312). The authors are grateful to Mrs. Chunxue Lu, Mrs. Lili Chen and Mr. Xiaoxing You for their excellent technical assistance and advice.

\section{References}

1. Braunstein M, Rajkumar P, Claus CL, Vaccarelli G, Moore AJ, Wang D and Anderson MK: HEBAlt enhances the T-cell potential of fetal myeloid-biased precursors. Int Immunol 22: 963-972, 2010 
2. van de Beek D, De Gans J, Spanjaard L, Weisfelt M, Reitsma JB and Vermeulen M: Clinical features and prognostic factors in adults with bacterial meningitis. N Engl J Med 351: 1849-1859, 2004

3. Harrison LH, Mohan N and Kirkpatrick P: Meningococcal group A, C, Y and W-135 conjugate vaccine. Nat Rev Drug Discov 9: 429-430, 2010

4. Khatami A and Pollard AJ: The epidemiology of meningococcal disease and the impact of vaccines. Expert Rev Vaccines 9: 285-298, 2010

5. Águeda S, Campos T and Maia A: Prediction of bacterial meningitis based on cerebrospinal fluid pleocytosis in children Braz J Infect Dis 17: 401-404, 2013.

6. Raymond J: Neisseria meningitidis: characterisation and epidemiology. Arch Pediatr 19 (Suppl 2): S55-S60, 2012 (In French).

7. Ferguson LE, Hormann MD, Parks DK and Yetman RJ: Neisseria meningitidis: presentation, treatment, and prevention. J Pediatr Health Care 16: 119-124, 2002.

8. Findlow J, Lowe A, Deane S, Balmer P, van den Dobbelsteen G, Dawson M, Andrews $\mathrm{N}$ and Borrow R: Effect of sequence variation in meningococcal PorA outer membrane protein on the effectiveness of a hexavalent PorA outer membrane vesicle vaccine in toddlers and school children. Vaccine 23: 2623-2627, 2005.

9. van den Dobbelsteen GP, van Dijken HH, Pillai S and van Alphen L: Immunogenicity of a combination vaccine containing pneumococcal conjugates and meningococcal PorA OMVs. Vaccine 25: 2491-2496, 2007.

10. Aaberge IS, Oster P, Helland OS, Kristoffersen AC, Ypma E, Høiby EA, Feiring B and Nøkleby H: Combined administration of meningococcal serogroup B outer membrane vesicle vaccine and conjugated serogroup $C$ vaccine indicated for prevention of meningococcal disease is safe and immunogenic. Clin Diagn Lab Immunol 12: 599-605, 2005.

11. Zhou H, Gao Y, Xu L, Li M, Li Q, Li Y, Liang X, Luo H, Kan B, $\mathrm{Xu} \mathrm{J}$ and Shao Z: Distribution of serogroups and sequence types in disease-associated and carrier strains of Neisseria meningitidis isolated in China between 2003 and 2008. Epidemiol Infect 140: 1296-1303, 2012

12. Yang L, Shao Z, Zhang X, Xu L, Peng J, Xu X, Liang X, Qi Y and Jin Q: Genotypic characterization of Neisseria meningitidis serogroup B strains circulating in China. J Infect 56: 211-218, 2008 .

13. Panatto D, Amicizia D, Lai PL and Gasparini R: Neisseria meningitidis B vaccines. Expert Rev Vaccines 10: 1337-1351, 2011.

14. Silva GP, Cruz SC, Cruz AC and Milagres LG: Short-term and long-term antibody response by mice after immunization against Neisseria meningitidis B or diphtheria toxoid. Braz J Med Biol Res 46: 148-153, 2013

15. Martin D, Cadieux N, Hamel J and Brodeur BR: Highly conserved Neisseria meningitidis surface protein confers protection against experimental infection. J Exp Med 185: 1173-1183, 1997.

16. Halperin SA, Langley JM, Smith B, Wunderli P, Kaufman L, Kimura A and Martin D: Phase 1 first-in-human studies of the reactogenicity and immunogenicity of a recombinant meningococcal NspA vaccine in healthy adults. Vaccine 25: 450-457, 2007

17. Vandeputte-Rutten L, Bos MP, Tommassen J and Gros P: Crystal structure of Neisserial surface protein A (NspA), a conserved outer membrane protein with vaccine potential. J Biol Chem 278: 24825-24830, 2003

18. Tsolakos N, Lie K, Bolstad K, Maslen S, Kristiansen PA, Hoiby EA, Wallington A, Vipond C, Skehel M, Tang CM, Feavers IM, Wedege E and Wheeler JX: Characterization of meningococcal serogroup B outer membrane vesicle vaccines from strain 44/76 after growth in different media. Vaccine 28: $3211-3218,2010$

19. Zimmer SM and Stephens DS: Serogroup B meningococcal vaccines. Curr Opin Investig Drugs 7: 733-739, 2006.
20. Tamargo B, Márquez Y, Ramírez W, Cedré B, Fresno M and Sierra G: New proteoliposome vaccine formulation from N. meningitidis serogroup $\mathrm{B}$, without aluminum hydroxide, retains its antimeningococcal protectogenic potential as well as Th-1 adjuvant capacity. BMC Immunol 14 (Suppl 1): S12, 2013.

21. Richmond P, Kaczmarski E, Borrow R, Findlow J, Clark S, McCann R, Hill J, Barker M and Miller E: Meningococcal C polysaccharide vaccine induces immunologic hyporesponsiveness in adults that is overcome by meningococcal $\mathrm{C}$ conjugate vaccine J Infect Dis 181: 761-764, 2000.

22. Cassataro J, Velikovsky CA, Bruno L, Estein SM, de la Barrera S, Bowden R, Fossati CA and Giambartolomei GH: Improved immunogenicity of a vaccination regimen combining a DNA vaccine encoding Brucella melitensis outer membrane protein 31 (Omp31) and recombinant Omp31 boosting. Clin Vaccine Immunol 14: 869-874, 2007.

23. Martin D, Brodeur BR, Hamel J, Couture F, de Alwis U, Lian Z, Martin S, Andrews D and Ellis RW: Candidate Neisseria meningitidis NspA vaccine. J Biotechnol 83: 27-31, 2000.

24. Cadieux N, Plante M, Rioux CR, Hamel J, Brodeur BR and Martin D: Bactericidal and cross-protective activities of a monoclonal antibody directed against Neisseria meningitidis NspA outer membrane protein. Infect Immun 67: 4955-4959, 1999.

25. Lewis LA, Ngampasutadol J, Wallace R, Reid JE, Vogel U and Ram S: The meningococcal vaccine candidate neisserial surface protein $\mathrm{A}(\mathrm{NspA})$ binds to factor $\mathrm{H}$ and enhances meningococcal resistance to complement. PLoS Pathog 6: e1001027, 2010.

26. Arenas J, Nijland R, Rodriguez FJ, Bosma TN and Tommassen $\mathrm{J}$ : Involvement of three meningococcal surface-exposed proteins, the heparin-binding protein NhbA, the $\alpha$-peptide of $\operatorname{IgA}$ protease and the autotransporter protease NalP, in initiation of biofilm formation. Mol Microbiol 87: 254-268, 2013.

27. Vaughan AT, Gorringe A, Davenport V, Williams NA and Heyderman RS: Absence of mucosal immunity in the human upper respiratory tract to the commensal bacteria Neisseria lactamica but not pathogenic Neisseria meningitidis during the peak age of nasopharyngeal carriage. J Immunol 182: 2231-2240, 2009.

28. Davenport V, Groves E, Horton RE, Hobbs CG, Guthrie T, Findlow J, Borrow R, Naess LM, Oster P, Heyderman RS and Williams NA: Mucosal immunity in healthy adults after parenteral vaccination with outer-membrane vesicles from Neisseria meningitidis serogroup B. J Infect Dis 198: 731-740, 2008.

29. Trotter CL, Yaro S, Njanpop-Lafourcade BM, Drabo A, Kroman SS, Idohou RS, Sanou O, Bowen L, Findlow H, Diagbouga S, Gessner BD, Borrow R and Mueller JE: Seroprevalence of bactericidal, specific IgG antibodies and incidence of meningitis due to group A Neisseria meningitidis by age in Burkina Faso 2008. PLoS One 8: e55486, 2013.

30. Zhao F, Wang S, Zhang X, Gu W, Yu J, Liu S, Zeng T, Zhang Y and Wu Y: Protective efficacy of a Treponema pallidum Gpd DNA vaccine vectored by chitosan nanoparticles and fused with interleukin-2. Can J Microbiol 58: 117-123, 2012.

31. Haghi F, Peerayeh SN, Siadat SD and Montajabiniat M: Cloning, expression and purification of outer membrane protein PorA of Neisseria meningitidis serogroup B. J Infect Dev Ctries 5: 856-862, 2011.

32. da Hora VP, Conceição FR, Dellagostin OA and Doolan DL: Non-toxic derivatives of LT as potent adjuvants. Vaccine 29 $1538-1544,2011$

33. Jung DJ, An JH, Kurokawa K, Jung YC, Kim MJ, Aoyagi Y, Matsushita M, Takahashi S, Lee HS, Takahashi K and Lee BL: Specific serum Ig recognizing staphylococcal wall teichoic acid induces complement-mediated opsonophagocytosis against Staphylococcus aureus. J Immunol 189: 4951-4959, 2012.

34. Kelly DF and Rappuoli R: Reverse vaccinology and vaccines for serogroup B Neisseria meningitidis. Adv Exp Med Biol 568: 217-223, 2005. 